Conclusion In the setting of acute severe ulcerative colitis, serum calprotectin is comparable with serum CRP in predicting outcome. Further work is needed to establish if it may be a useful predictor of outcome in patients with ulcerative colitis who fail to mount a high CRP response despite endoscopic assessment confirming severe active inflammation. Work is also ongoing to establish its utility in the outpatient setting both in Crohn's disease and ulcerative colitis. Disclosure of Interest None Declared.

\section{PTH-083 METHYLATION SIGNATURES OF NON-EXPRESSED GENES REVEAL INSIGHTS INTO THE EFFECTS OF INFLAMMATION ON STEM CELL DYNAMICS AND CRYPT FISSION IN INFLAMIMATORY BOWEL DISEASE}

doi:10.1136/gutjnl-2013-304907.570

1." N Jawad, ${ }^{2} \mathrm{~T}$ Graham, ${ }^{3} \mathrm{M}$ Novelli, ${ }^{3} \mathrm{M}$ Rodriguez-Justo, ${ }^{1} \mathrm{~N}$ Wright, ${ }^{1} \mathrm{~S}$ McDonald. ${ }^{\prime} \mathrm{Cen}-$ tre for Tumour Biology, Barts Cancer Institute, Barts and The London School of Medicine and Dentistry, Queen Mary University of London, London, UK; ${ }^{2}$ Centre for Evolution and Cancer, University of California at San Francisco, San Francisco, United States, ${ }^{3}$ Histopathology Department, University College Hospital, London, UK

Introduction Inflammatory bowel disease (IBD) confers a high risk of development of colitis-associated colorectal cancer (CACRC) in patients with extensive colitis. Crypt fission (a crypt bifurcating into two) has been shown to be a mechanism of clonal expansion in the intestinal epithelium. Although fission is rare in normal colon, many crypts in patients with colitis appear to be in the process of fission. A recent study from the host laboratory demonstrated that protumourigenic mutations can spread through the entire inflamed colon suggesting that this occurs at a considerable rate indicating stem cell dynamics are altered in $\mathrm{IBD}^{1}$

Methods Somatic mitochondrial DNA (mtDNA) mutations are a reliable marker of clonal expansion in human colon. Combining mtDNA mutations with additional markers of clonal expansion that change over time, such as methylation patterns of nonexpressed genes, reveals whether populations of cells show a recent ancestry. This is measured by evaluating methylation pattern diversity between samples. Methylation patterns of CSX and MYOD1 genes were examined in clonally related and unrelated crypts from multiple areas in IBD patients by laser capture microdissection bisulphite sequencing. Clonality was demonstrated by cytochrome c oxidase deficient (CCO-) cells sharing an identical somatic mtDNA mutation.

Results In active inflammation, both adjacent clonally related $\mathrm{CCO}$ - crypts and adjacent unrelated crypts had similar methylation patterns, indicating recent crypt fission. In contrast, adjacent unrelated crypts in quiescent disease had dissimilar methylation patterns, indicating that crypt fission rates are slow and resemble that of normal colon. The number of unique methylation patterns in crypts from active IBD were significantly less than those obtained from normal patients suggesting that niche succession (a stem cell populating the niche) is elevated in IBD.

Conclusion Elevated crypt fission in active IBD may explain the extensive dispersion of protumourigenic clones previously observed in IBD. Subsequent cycles of crypt atrophy and mucosal healing by crypt fission, may provide a key growth stimulus in the inflamed colon. Furthermore, there appears to be an increased rate at which a single stem cell populates the niche within IBD crypts. Such expansion facilitates the establishment of protumourgenic mutations within crypts.

Disclosure of Interest None Declared.

\section{REFERENCE}

1. Galandiuk S, Rodriguez-Justo M, Jeffery R, Nicholson AM, Cheng Y, Oukrif D, Elia G, Leedham SJ, McDonald SA, Wright NA et al. 2012. Field cancerization in the intestinal epithelium of patients with Crohn's ileocolitis. Gastroenterology 142: 855-864 e858

\section{PTH-084 ENDOSCOPIC AND HISTOLOGICAL ACTIVITY AS PREDICTORS OF RELAPSE IN PATIENTS UNDERGOING SURVEILLANCE COLONOSCOPY FOR ULCERATIVE COLITIS}

doi:10.1136/gutjnl-2013-304907.571

1.2, $\mathrm{N}$ Mohammed, ${ }^{1} \mathrm{C}$ F Donnellan, ' S M Everett, ${ }^{1,2} \mathrm{~A}$ C Ford, ${ }^{1,2} \mathrm{P} \mathrm{J}$ Hamlin, ' $\mathrm{J}$ S Jennings, 'B J Rembacken, 'A Sainsbury, ${ }^{1}{ }^{12} \mathrm{~V}$ Subramanian. ${ }^{1}$ Department of Gastroenterology, Leeds teaching hospitals NHS Trust; ${ }^{2}$ Department of Gastroenterology, Leeds Institute of Molecular Medicine, Leeds, UK

Introduction Mucosal healing has shown to correlate with improved long term outcomes in patients with inflammatory bowel disease. Histological inflammation is often noted in endoscopically normal mucosa. We aimed to investigate the predictive role of endoscopic and histological inflammation on disease relapse in UC patients in clinical remission

Methods We conducted a retrospective review of adult patients in clinical remission who underwent surveillance colonoscopies in our institution from January 2008 to December 2011. Electronic records were reviewed for endoscopy reports and subsequent clinical care. Data was recorded on age, gender, duration and extent of the disease, medications, steroid use in the last 6 months, Mayo endoscopic score, Geboes histological activity index and follow up data for any flares till date. Patients were deemed to have a relapse if they required steroids or an increase in their medication dose fro symptom control in the subsequent 12 months following their index colonoscopy.

Statistical analysis: Rate of clinical relapse and the predictive value of the variables of interest were assessed using SPSS version 17. All variable analysed in univariate fashion and included in multivariate analysis if $\mathrm{p}$ was less than $\leq 0.3$. Multivariate analysis was based on an automated backward logistic selection process. P values $<0.05$ were considered significant.

Results 406 patients underwent surveillance colonoscopy during the study period of which 317 (Male: 172 Females: 145) met the inclusion criteria. 57 patients (Males 29, females 28) relapsed within 12 months (Table 1 provides the baseline characteristics). On univariate analysis age (OR $0.9695 \%$ CI $0.94-0.98$ ), Geboes score $\geq 2$ $(4.53,2.40-8.52)$ and Mayo score $\geq 1(3.72,2.05-6.73)$ were significantly associated with relapse. Duration of disease $(p=0.09)$, use of immunomodulators $(p=0.29)$ and recent steroid use $(p=0.3)$ were included in the multivariate analysis. On multivariate analysis Geboes score of $\geq 2(5.11,2.73-9.59)$ and age $(0.97,0.97-0.99)$ were predictive of clinical relapse.

\begin{tabular}{|c|c|c|}
\hline Variables & Flare up & No flare \\
\hline Age (mean $\pm S D)$ & $51.2 \pm 14.6$ & $57.9 \pm 12.5$ \\
\hline Endoscopic score $\leq 1$ & 22 & 182 \\
\hline Endoscopic score $\geq 2$ & 35 & 78 \\
\hline Geboes score $<2$ & 33 & 224 \\
\hline Geboes score $>2$ & 24 & 36 \\
\hline
\end{tabular}

Conclusion Histological activity and younger age are significant predictors of disease relapse in patients undergoing surveillance endoscopy. Endoscopic activity with standard white light endoscopy did not predict clinical relapse. Better non-invasive markers of disease relapse are required for patients with ulcerative colitis Disclosure of Interest None Declared.

PTH-085 MONOCLONAL ANTIBODY THERAPY IN CROHN'S DISEASE: DOES SERIAL FAECAL CALPROTECTIN PLAY A ROLE?

doi:10.1136/gutjnl-2013-304907.572

${ }^{1} \mathrm{~A}$ A Thi, 1,"P BASUMANI, ${ }^{2} \mathrm{M}$ Walsh, 'M Jerrison, ${ }^{1} \mathrm{~K} \mathrm{D}$ Bardhan. 'Gastroenterology, Rotherham General Hospital NHS TRUST, Rotherham; 'astroenterology, Rotherham General Hospital NHS TRUST, Rrotherham, UK 